\title{
Food intake patterns associated with carotid artery atherosclerosis in the Insulin Resistance Atherosclerosis Study
}

\author{
Angela D. Liese ${ }^{1} *$, Michele Nichols ${ }^{1}$, Denise Hodo ${ }^{1}$, Philip B. Mellen ${ }^{2}$, Mandy Schulz ${ }^{3}$, David C. Goff $\mathrm{Jr}^{4}$ \\ and Ralph B. D'Agostino $\mathrm{Jr}^{4}$ \\ ${ }^{1}$ Department of Epidemiology and Biostatistics and Center for Research in Nutrition and Health Disparities, Arnold School of \\ Public Health, University of South Carolina, 800 Sumter Street, Columbia, SC 29208, USA \\ ${ }^{2}$ Hypertension Center, Hattiesburg Clinic, Hattiesburg, MS, USA \\ ${ }^{3}$ Department of Epidemiology, German Institute of Human Nutrition Potsdam-Rehbruecke, Nuthetal, Germany \\ ${ }^{4}$ Division of Public Health Sciences, Wake Forest University School of Medicine, Winston-Salem, NC, USA
}

(Received 22 June 2009 - Revised 27 October 2009 - Accepted 11 November 2009 - First published online 22 January 2010)

We aimed to identify food intake patterns that operate via haemostatic and inflammatory pathways on progression of atherosclerosis among 802 middle-aged adults with baseline and 5-year follow-up ultrasound measurements of common (CCA) and internal carotid artery (ICA) intimal medial thickness (IMT). Food intake was ascertained with an FFQ. We derived food patterns using reduced rank regression (RRR) with plasminogen activator inhibitor 1 and fibrinogen as response variables. We explored the impact of various food pattern simplification approaches. We identified a food pattern characterised by higher intakes of less healthful foods (low-fibre bread and cereal, red and processed meat, cottage cheese, tomato foods, regular soft drinks and sweetened beverages) and lower intakes of more healthful foods (wine, rice and pasta, meal replacements and poultry). The pattern was positively associated with mean CCA IMT at follow-up $(P=0.0032)$, a $1 \mathrm{SD}$ increase corresponding to an increase of $13 \mu \mathrm{m}$ higher CCA IMT at follow-up, adjusted for demographic and cardiovascular risk factors. With increasing pattern quartile (Q), the percentage change in CCA IMT increased significantly: Q1 0.8\%; Q2 3.2\%; Q3 8.6\%; Q4 7.9\% (P=0.0045). No clear association with ICA IMT was observed. All simplification methods yielded similar results. The present results support the contention that a pro-inflammatory and pro-thrombotic dietary pattern increases the rate of coronary artery atherosclerosis progression, independent of traditional cardiovascular risk factors. RRR is a promising and robust tool for moving beyond the previous focus on nutrients or foods into research on the health effects of broader dietary patterns.

Dietary patterns: Inflammation: Haemostasis: Nutrition: Atherosclerosis

Reduced rank regression (RRR) is increasingly recognised as a promising method in dietary pattern research. Food intake patterns are identified among food groups by concurrently utilising data on a set of response variables, ideally biomarkers, selected because of known associations with the disease of interest $^{(1)}$. RRR can be particularly powerful if the response factors are highly predictive biomarkers.

Interestingly, only a few studies to date have applied RRR to the evaluation of dietary predictors of CHD, even though this field would seem particularly fruitful given the well-established characterisation of biomarker risk factors for CHD. In one such endeavour, homocysteine metabolism-related biomarkers were utilised and the investigators found a strong inverse association between a healthful food intake pattern and $\mathrm{CHD}^{(2)}$. Two studies utilising either a range of biomarkers including lipids, C-reactive protein and C-peptide ${ }^{(3)}$ or focusing on lipid levels ${ }^{(4)}$ identified largely detrimental food intake patterns strongly associated with CHD.
To the best of our knowledge, neither prospective studies employing RRR have been conducted that have used multiple markers of inflammation nor has any study focused on markers of subclinical atherosclerosis. Carotid intimal medial thickness (IMT) is now an established marker for early atherosclerosis and has been shown to be a strong predictor of future vascular events ${ }^{(5)}$. A 1 SD common carotid artery (CCA) IMT difference has been associated with a relative risk of myocardial infarction of 1.26 , or in absolute terms, a $0.10 \mathrm{~mm}$ CCA IMT difference has been associated with a relative risk of $1 \cdot 15^{(5)}$. Furthermore, there is now consistent evidence that fibrinogen ${ }^{(6)}$ is a risk factor for CVD. The association of plasminogen activator inhibitor 1 (PAI-1) with CHD risk is suggestive, but likely more complex ${ }^{(7,8)}$.

The purpose of the present study was to identify RRRdetermined food intake patterns that affect inflammatory biomarkers and to evaluate their association with progression of carotid artery atherosclerosis in the prospective Insulin

Abbreviations: CCA, common carotid artery; FS, full food pattern score; ICA, internal carotid artery; IMT, intimal medial thickness; IRAS, Insulin Resistance

Atherosclerosis Study; PAI-1, plasminogen activator inhibitor 1; RRR, reduced rank regression; SI, insulin sensitivity.

* Corresponding author: Angela D. Liese, fax +1803777 2524, email liese@sc.edu 
Resistance Atherosclerosis Study (IRAS). We additionally aimed to address a methodological question on how best to translate food intake patterns identified by RRR to practically useful patterns and explored the impact of a variety of food pattern simplification approaches on our findings.

\section{Research design and methods}

\section{Study population}

IRAS is a multi-centre, observational study evaluating the relations between insulin resistance, cardiovascular risk factors and disease in a multi-ethnic cohort ${ }^{(9)}$. The study was designed to obtain nearly equal representation of participants across age, sex, three race/ethnic groups (non-Hispanic White, Black and Hispanic) and glucose tolerance status (normal, impaired glucose tolerance and non-insulin taking type 2 diabetes mellitus) and sampled from four clinical centres (Los Angeles, CA, Oakland, CA, San Luis Valley, CO and San Antonio, TX, USA). The present study was conducted according to the guidelines laid down in the Declaration of Helsinki and all procedures involving human subjects were approved by the respective field centre's institutional review boards (University of Colorado Health Sciences Center, University of Texas Health Sciences Center, University of Southern California School of Medicine, and Kaiser Permanente, CA, USA). Written informed consent was obtained from all subjects.

A total of 1624 participants ages 40-69 were recruited for the baseline examination (1992-4), which included a baseline history, physical examination, carotid ultrasonography and laboratory measures and was conducted in two visits. In 1997-9, the cohort was invited to a follow-up examination under an identical protocol (average follow-up $5 \cdot 2$ years) to which $81 \%$ returned.

For this analysis, only the 1088 adults with normal or impaired glucose tolerance at baseline, of whom $907(83 \%)$ returned to follow-up, were eligible because we were concerned that persons with diabetes may have changed their diet following diagnosis. Note that no differences in race/ ethnicity, clinic, sex or age were observed between those lost to follow-up and those who returned, except a slightly lower educational attainment in those lost to follow-up. We subsequently excluded forty participants with missing baseline or follow-up data on CCA and internal carotid artery (ICA) IMT ( $n$ 867). After excluding sixty-five persons with missing baseline data on dietary intake, PAI-1 and fibrinogen, we were left with a total of 802 observations with complete data. Data completeness was unrelated to demographic variables (age, sex, race and education), except study centre.

\section{Assessment of dietary intake and independent variables}

Each examination required two visits conducted approximately 1 week apart. Participants had fasted for $12 \mathrm{~h}$, abstained from heavy exercise and alcohol for $24 \mathrm{~h}$ and refrained from smoking in the morning of both visits. At the first visit day, after an initial blood sample, a $2 \mathrm{~h}, 75 \mathrm{~g}$ oral glucose tolerance test (Orange-dex; Custom Laboratories, Baltimore, MD, USA) was performed. Before the oral glucose tolerance test, a blood sample was taken for the assessment of a variety of laboratory parameters including the biomarkers used in this analysis, PAI-1 and fibrinogen. The interviews and other anthropometric measurements were also conducted on this day. At the second visit, a twelve sample, insulin-enhanced, frequently sampled intravenous glucose tolerance test ${ }^{(10,11)}$ was conducted. Insulin sensitivity $\left(S_{\mathrm{I}}\right)$ and acute insulin response were assessed using minimal model analysis ${ }^{(12,13)}$. Acute insulin response was calculated based on insulin levels through the $8 \mathrm{~min}$ blood samples before insulin infusion. Diabetes was defined as $2 \mathrm{~h}$ glucose $\geq 2000 \mathrm{mg} / \mathrm{l}$, according to the 1985 WHO criteria ${ }^{(14)}$, or under hypoglycaemic medication.

PAI-1 was measured in citrated plasma with a two-site immunoassay that is sensitive to free active and latent PAI-1 but not to PAI-1 complexed with tissue plasminogen activator. Details of plasma processing have been described ${ }^{(15)}$. The CV was $14 \%$. Fibrinogen was measured in citrated plasma with a modified clot-rate assay, using the Diagnostica STAGO ST4 instrument (Diagnostica, Inc., Parsippany, NJ, USA) with a $\mathrm{CV}$ of $3.0 \%$. Plasma lipid concentrations were determined at the central IRAS laboratory using the Lipid Research Clinics methodology.

Height, waist and weight were measured in duplicate and recorded to the nearest $0.5 \mathrm{~cm}$ and $0.1 \mathrm{~kg}$, respectively. BMI was calculated as weight $/$ height $^{2}\left(\mathrm{~kg} / \mathrm{m}^{2}\right)$. Blood pressure was measured three times $5 \mathrm{~min}$ apart with a $\mathrm{Hg}$ sphygmomanometer and the participant in a sitting position. The second and third measurements were averaged and utilised. Hypertension was defined as systolic blood pressure $140 \mathrm{mmHg}$ and/or diastolic blood pressure $90 \mathrm{mmHg}$ or under current use of antihypertensive medication.

Usual dietary intake was assessed using a 1-year, semi-quantitative, interviewer administered 114-item FFQ modified from the National Cancer Institute Health History and Habits Questionnaire to include regional and ethnic food choices across the four clinical centres ${ }^{(16)}$. Participants were asked to recall intake of foods and beverages over the past year including frequency and serving size. The FFQ was administered on the first visit day by centrally trained and certified interviewers. For analyses, the servings per day were standardised to the medium serving size by multiplying the intake frequency with the serving size after applying a weighting factor $($ small $=0 \cdot 5$, medium $=1 \cdot 0$ and large $=1 \cdot 5)$. From the 114 line items of the FFQ, we created thirty-three food groups based on similarities in food and nutrient composition as described $^{(17)}$. Subjects were asked separately about their usual consumption of alcoholic beverages. Daily nutrient and energy intake was estimated from the FFQ and the alcohol questionnaire using an expanded nutrient database (HHHQDIETSYS analysis software, version 3.0; NCI, Bethesda, MD, USA, 1993). The IRAS FFQ was validated in a subset of the IRAS population among whom eight $24 \mathrm{~h}$ dietary recalls were collected by telephone followed by an administration of the $\mathrm{FFQ}^{(16)}$. Validity was assessed by comparing dietary intake values, including supplements, obtained from the FFQ to the average intake estimated from the $24 \mathrm{~h}$ recalls. Pearson correlation coefficients adjusted for total energy were 0.44 for total fat and 0.37 for total carbohydrates with a mean correlated across all nutrients of 0.50 unadjusted and 0.47 energy adjusted.

A structured interview was used to collect 1-year recall of physical activity from which total energy expenditure was 
estimated $^{(18)}$. Family history of diabetes, smoking status and race/ethnicity were self-reported.

\section{Carotid ultrasonography}

Participants underwent high-resolution B-mode carotid ultrasonography at baseline and 5-year follow-up using identical scanning protocols and equipment. The protocol included bilateral assessment of wall thickness for the CCA and ICA. For ICA, the sonographer identified the site of maximal thickness between the dilatation of the carotid bulb and the region of the ICA above the carotid bifurcation. It should be noted that carotid wall thickness represents the sum of the intima and media sections of the arterial wall; hence, greater IMT may represent varying mixtures of thickening due to atherosclerosis, confined primarily to the intima, and other processes that might cause thickening, perhaps via smooth muscle proliferation, in the media. Because atherosclerotic plaque is uncommon in the CCA, the CCA wall thickness can be interpreted as arterial wall changes in the absence of raised atherosclerotic plaque. In contrast, thickening of the ICA and the carotid bifurcation are more likely due to the fatty and fibrous accumulation of plaque which needs to be considered in the interpretation of the ICA wall thickness ${ }^{(19)}$. Three images were taken bilaterally at different interrogation angles (proximal, lateral and anterior). For the CCA, bilateral images were taken $1 \mathrm{~cm}$ proximal to the dilatation of the carotid bulb at a single (lateral) angle. At follow-up, the sonographer sought the site of maximal IMT, independent of its location at baseline.

Ultrasound images were recorded on super-video home system tape and read at a central facility after completion of the follow-up examination by a single reader blinded to participant, participant characteristics and examination. For each of the eight available images, the maximal IMT was taken over a $1 \mathrm{~cm}$ segment of the arterial wall distant from the skin surface (far wall). Two summary measures were calculated: (1) the mean of the six ICA sites; (2) the mean of the two CCA sites. To allow equal weighting of the left and right arteries in the presence of missing data, the mean value of the available measures on the left ICA and the mean values of the available measures on the right ICA were calculated and then the mean of these two means was used in analysis. This approach is identical or similar to that used in other epidemiological studies of atherosclerosis ${ }^{(20,21)}$ and clinical trials ${ }^{(22)}$.

\section{Statistical analysis}

RRR, also known as maximum redundancy analysis, was employed to identify food patterns by using the SAS PLS procedure with the method $=$ RRR option ${ }^{(1)}$. Because the RRR procedure is not well known, we first explain the terminology specific to this method. We distinguish two types of observed variables, namely the food groups which are used as predictor variables and the biomarkers PAI-1 and fibrinogen which are used as response variables. RRR produces two sets of scores, $X$ and $Y$ scores. Note that RRR is similar to factor analysis in its mathematical foundation but applied to two sets of observed variables concurrently. To conceptualise what RRR does, it might be useful to think of it as a principal component analysis applied to responses and a subsequent linear regression of principal components on predictors although it is somewhat more sophisticated and efficient than this twostep procedure. RRR extracts food pattern scores ( $X$ scores), which are linear functions from predictor variables (i.e. food groups, here thirty-three groups as described in Liese et al. ${ }^{(17)}$ ) while concurrently maximising the explained variation in a set of response variables (i.e. PAI-1 and fibrinogen). In RRR, the coefficient vectors of the extracted linear functions are eigenvectors of a covariance matrix. Thereby, a response score (i.e. $Y$ score) is created, which is then projected onto the space of predictors (foods) forming a food pattern score. Response and food pattern score reflect the same unobserved or latent variable in different sets of original variables. Because the number of extracted food pattern scores is equal to the number of response variables, two scores were originally identified by RRR. Only the first pattern score (referred to as the original score) was retained for subsequent analyses, and results shown in Tables 1-4 pertain exclusively to this first pattern score. The second pattern score was not associated with IMT and results are shown in the online appendix.

To reduce the dependency of the score from the data, we simplified the original score by regressing the standardised food groups on the $Y$ score. Only those food groups that were predictive at the level of $\alpha=0.05$ were retained. These standardised food groups were then summed, while retaining the direction of the factor loading ${ }^{(23)}$. This method is referred to as M1.

Given that several other simplification approaches have been proposed ${ }^{(3,24)}$, we have evaluated whether the choice of method may impact the present results. Two variations were evaluated selecting: (1) ten food groups explaining the largest amount of variation in $X$ score (method 2, M2); (2) five positively and five inversely associated food groups which explained the largest amount of variation in $X$ score $(\mathrm{M} 3)^{(3)}$. Note that the explained variation was calculated by multiplying the standardised score parameters (obtained from regressing the food groups on the pattern score) with the correlation coefficients (empirical Pearson correlation coefficient of the individual food groups with the original score) and 100 . We have also applied a commonly used simplification approach selecting only food groups with high factor loadings $\geq|0 \cdot 2|$ (method 4, M4), which were estimated within the RRR procedure and represent the correlation of the food group with the original (non-simplified) score ${ }^{(24)}$.

To evaluate the relation of the simplified food pattern score to carotid atherosclerosis over time, we examined three different outcomes in linear regression models including: (1) the mean IMT at follow-up (adjusted for baseline IMT); (2) the change in IMT (follow-up IMT - baseline IMT); (3) the percentage change in IMT (change in IMT divided by baseline IMT $\times 100)$. Each model was adjusted for age, sex, race/ethnicity, clinic, glucose tolerance status at baseline, smoking, systolic and diastolic blood pressure, BMI and total and HDL-cholesterol. We present estimates of mean outcome level by score quartiles based on least square means obtained from each of the models. The test for trend across quartiles of the food pattern scores used the $P$ value resulting from the type III analysis of effects contrast for linear trend. 
Table 1. Food groups strongly associated with food intake pattern and their characteristics by pattern score quartile $(n 867)$ (Mean values and standard deviations)

\begin{tabular}{|c|c|c|c|c|c|c|c|c|c|c|c|c|c|}
\hline \multirow[b]{3}{*}{ Food group } & \multirow{2}{*}{\multicolumn{4}{|c|}{ Characteristics of original food pattern score ${ }^{*}$}} & \multicolumn{8}{|c|}{ Quartiles of simplified $\dagger$ food pattern score (servings/d) } & \multirow[b]{3}{*}{$\begin{array}{c}P \\
\text { for trend }\end{array}$} \\
\hline & & & & & \multicolumn{2}{|c|}{1} & \multicolumn{2}{|c|}{2} & \multicolumn{2}{|c|}{3} & \multicolumn{2}{|c|}{4} & \\
\hline & $\begin{array}{l}\text { Factor } \\
\text { loading }\end{array}$ & $\begin{array}{l}\text { Standard score } \\
\text { parameter }\end{array}$ & $\begin{array}{l}\text { Pearson's correlation } \\
\text { coefficient§ }\end{array}$ & $\begin{array}{l}\text { Explained variation } \\
\text { in score } \|\end{array}$ & Mean & SD & Mean & SD & Mean & SD & Mean & SD & \\
\hline Low-fibre bread/cereal** ${ }^{\star *}$ & 0.42 & 0.33 & 0.64 & $21 \cdot 20$ & 0.6 & 0.5 & 0.8 & 0.6 & 1.4 & 0.8 & $2 \cdot 2$ & $1 \cdot 2$ & $<0.0001$ \\
\hline Red and processed meat $\dagger$ & 0.41 & 0.26 & 0.63 & $16 \cdot 10$ & 0.5 & 0.4 & 0.7 & 0.5 & 1.0 & 0.6 & 1.7 & 0.8 & $<0.0001$ \\
\hline Cottage cheesełł & 0.21 & 0.29 & 0.33 & $9 \cdot 35$ & 0.02 & 0.05 & 0.05 & 0.09 & 0.07 & $0 \cdot 10$ & 0.14 & 0.23 & $<0.0001$ \\
\hline Tomato foods§§ & 0.24 & 0.24 & 0.37 & 8.88 & 0.5 & 0.4 & 0.6 & 0.5 & 0.8 & 0.5 & 1.0 & 0.6 & $<0.0001$ \\
\hline Regular soft drinks \|\| & $0 \cdot 19$ & 0.20 & 0.28 & 5.63 & $0 \cdot 1$ & 0.2 & 0.1 & 0.3 & 0.2 & 0.3 & 0.4 & 0.4 & $<0.0001$ \\
\hline Wineๆ ๆ & -0.21 & -0.28 & -0.32 & $9 \cdot 14$ & 0.4 & 0.8 & 0.1 & 0.3 & $0 \cdot 1$ & 0.2 & 0.0 & $0 \cdot 1$ & $<0.0001$ \\
\hline Rice and pasta ${ }^{* \star *}$ & -0.11 & -0.27 & -0.16 & 4.33 & 0.7 & 0.6 & 0.6 & 0.5 & 0.5 & 0.4 & 0.5 & 0.4 & $<0.0001$ \\
\hline Meal replacements†t† & -0.15 & -0.18 & -0.22 & 3.99 & $0 \cdot 1$ & 0.3 & 0.0 & 0.1 & 0.0 & 0.0 & 0 & 0 & $<0.0001$ \\
\hline 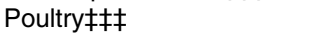 & -0.12 & -0.17 & -0.17 & 2.93 & 0.6 & 0.5 & 0.4 & 0.3 & 0.3 & 0.3 & 0.3 & 0.3 & $<0.0001$ \\
\hline
\end{tabular}

*The food pattern score presented here is the first score obtained in the reduced rank procedure.

† Simplification conducted using method 1 (stepwise linear regression on $Y$ score, $\alpha=0.05$ criteria).

¥ Standardised score parameters obtained from multiple linear regression of food groups on original food pattern score.

$\S$ Pearson's correlation coefficient obtained from correlating individual food groups with the original food pattern score.

\| Explained variation is calculated by multiplying the standardised parameters with the correlation coefficients and 100 .

I Values in parentheses are weighting factors applied to the number of servings/d for items contributing to more than one food group or for items contributing different proportions to one food group according to their actual consumption.

** Low-fibre bread and cereal: white bread, biscuits, starch and maize tortilla, maize bread, fortified cereal, cold cereal, sweetened cereal, cooked cereal (0.5), pizza, burritos, enchiladas and tacos.

††Red and processed meat: hamburgers, cheeseburgers, meat loaf, picadillo, carne guisada (asada); beef (steaks, roasts, etc. including on sandwiches); beef stew or pot pie with carrots or other vegetables (0.5); pork, including chops, roasts, or ribs; ham, ham hocks (including ham on sandwiches); game, including venison, rabbit; liver, including chicken livers; burritos, including breakfast burritos, soft taco with starch tortillas; green chilli con carne; Asian food; liverwurst; hot dogs (include pork, beef, turkey); bologna, salami, spam, other lunch meats (excluding ham); bacon; sausage, chorizo; veal, lamb; Italian sausage; pate.

¥¥Cottage cheese: cottage cheese, ricotta cheese.

$\S \S$ Tomato foods: tomatoes, tomato juice including chopped tomatoes and onion as condiments; salsa picante, taco sauce (0.5); spaghetti, lasagna, other pasta or mixed dishes with tomatoes or tomato sauce; pizza (0.5); enchiladas, tamales, tacos, tostades, chalupas, other Mexican dishes with maize tortillas (0.5); vegetable and tomato soup (0.5).

IIII Regular soft drinks (including colas, 7-up, etc.); lemonade, sweetened mineral water.

११ Wine includes both red and white wine.

${ }^{\star \star \star}$ Rice and pasta includes plain rice; pasta, noodles, fideo, couscous (without cheese or tomato sauce); spaghetti, lasagna, other pasta or mixed dishes with tomatoes or tomato sauce; macaroni and cheese; Asian food; sushi.

t†t Meal replacements are instant breakfasts and other liquid meals.

$\ddagger \ddagger \ddagger$ Poultry includes chicken, turkey or wild fowl (roasted, broiled or ground, incl. on sandwiches); chicken or turkey stew or pot pie with carrots or other vegetables; fried chicken; duck. 
Table 2. Study population characteristics by quartile of food intake pattern score ( $n$ 802)

(Mean values and standard deviations)

\begin{tabular}{|c|c|c|c|c|c|c|c|c|c|}
\hline & \multicolumn{8}{|c|}{ Quartiles of simplified ${ }^{\star}$ food pattern score } & \multirow[b]{3}{*}{$P$ for trend } \\
\hline & \multicolumn{2}{|c|}{1} & \multicolumn{2}{|c|}{2} & \multicolumn{2}{|c|}{3} & \multicolumn{2}{|c|}{4} & \\
\hline & Mean & SD & Mean & SD & Mean & SD & Mean & SD & \\
\hline \multicolumn{10}{|c|}{ Demographics and design characteristics } \\
\hline Age (years) & 54.9 & 8.7 & $55 \cdot 2$ & $8 \cdot 2$ & 54.5 & 8.4 & 53.9 & 8.4 & \\
\hline Male (\%) & 44.4 & & $42 \cdot 4$ & & $37 \cdot 8$ & & $49 \cdot 3$ & & \\
\hline Female (\%) & 55.6 & & $57 \cdot 6$ & & $62 \cdot 2$ & & $50 \cdot 7$ & & \\
\hline Non-Hispanic White (\%) & 44.9 & & $45 \cdot 2$ & & $42 \cdot 9$ & & $27 \cdot 7$ & & \\
\hline Non-Hispanic Black (\%) & $49 \cdot 1$ & & 35.9 & & $13 \cdot 8$ & & 5.5 & & \\
\hline Hispanic (\%) & $6 \cdot 0$ & & 18.9 & & $43 \cdot 3$ & & $66 \cdot 8$ & & \\
\hline Normal glucose tolerance (\%) & $71 \cdot 3$ & & $61 \cdot 8$ & & $66 \cdot 4$ & & $67 \cdot 3$ & & \\
\hline Impaired glucose tolerance (\%) & $28 \cdot 7$ & & 38.3 & & 33.6 & & $32 \cdot 7$ & & \\
\hline \multicolumn{10}{|l|}{ Biomarkers } \\
\hline PAl-1 (ng/ml) & $14 \cdot 1$ & $13 \cdot 7$ & 20.5 & 20.5 & $25 \cdot 1$ & $26 \cdot 7$ & $29 \cdot 4$ & $20 \cdot 2$ & $<0.0001$ \\
\hline Fibrinogen (mg/l) & 2715 & 555 & 2780 & 600 & 2707 & 497 & 2812 & 572 & 0.1972 \\
\hline Waist $(\mathrm{cm})$ & 88.5 & $12 \cdot 4$ & 89.5 & $12 \cdot 7$ & $90 \cdot 4$ & $12 \cdot 1$ & $92 \cdot 8$ & $13 \cdot 7$ & 0.0004 \\
\hline $\mathrm{BMI}\left(\mathrm{kg} / \mathrm{m}^{2}\right)$ & $27 \cdot 8$ & $5 \cdot 3$ & $28 \cdot 2$ & 5.4 & 28.5 & $5 \cdot 4$ & 29.1 & 6.4 & 0.0142 \\
\hline Fasting insulin (pmol/l) & 100 & 99 & 108 & 77 & 112 & 94 & 132 & 145 & 0.0025 \\
\hline$S_{l}\left(\min ^{-1} \times \mu U^{-1}\right.$ per $\left.\mathrm{ml} \times 10^{-4}\right)$ & $2 \cdot 6$ & $2 \cdot 2$ & $2 \cdot 1$ & 1.7 & $2 \cdot 1$ & $2 \cdot 2$ & $2 \cdot 0$ & 1.9 & 0.0051 \\
\hline AIR $(\mu \mathrm{U} / \mathrm{ml}$ per min $)$ & 54.8 & $46 \cdot 8$ & 63.0 & $46 \cdot 7$ & 65.4 & 44.5 & 79.4 & $77 \cdot 7$ & $<0.0001$ \\
\hline $\mathrm{TAG}(\mathrm{mmol} / \mathrm{l})$ & $113 \cdot 3$ & $64 \cdot 2$ & $129 \cdot 9$ & $79 \cdot 6$ & $139 \cdot 6$ & $93 \cdot 1$ & $151 \cdot 6$ & $102 \cdot 5$ & $<0.0001$ \\
\hline Total cholesterol $(\mathrm{mmol} / \mathrm{l})$ & $215 \cdot 2$ & 34.6 & $209 \cdot 6$ & $39 \cdot 7$ & 206.5 & 34.5 & $201 \cdot 3$ & $57 \cdot 6$ & 0.1755 \\
\hline HDL-cholesterol (mmol/l) & $51 \cdot 6$ & $16 \cdot 1$ & $48 \cdot 2$ & $15 \cdot 6$ & $44 \cdot 7$ & 13.5 & $42 \cdot 9$ & $13 \cdot 8$ & $<0.0001$ \\
\hline Systolic blood pressure $(\mathrm{mmHg})$ & $122 \cdot 4$ & $17 \cdot 5$ & $123 \cdot 2$ & $17 \cdot 6$ & $120 \cdot 9$ & $16 \cdot 3$ & $121 \cdot 2$ & $16 \cdot 9$ & 0.2783 \\
\hline Diastolic blood pressure $(\mathrm{mmHg})$ & 76.9 & 9.8 & 78.0 & 8.8 & $77 \cdot 2$ & $9 \cdot 0$ & 78.9 & $9 \cdot 1$ & 0.0583 \\
\hline \multicolumn{10}{|l|}{ Outcomes } \\
\hline CCA IMT $(\mu \mathrm{m})$ & 0.84 & 0.18 & 0.85 & 0.22 & 0.85 & 0.31 & 0.81 & 0.24 & 0.1620 \\
\hline CCA 5-year change $(\mu \mathrm{m})$ & 0.01 & 0.26 & 0.02 & 0.15 & 0.06 & 0.26 & 0.03 & 0.16 & 0.0841 \\
\hline CCA percentage change (\%) & 3.35 & 18.02 & 4.02 & $16 \cdot 28$ & 7.37 & 33.46 & 5.44 & $19 \cdot 66$ & 0.1761 \\
\hline ICA IMT $(\mu \mathrm{m})$ & 1.02 & 0.59 & 1.08 & 0.60 & 0.99 & 0.52 & 0.94 & 0.46 & 0.0382 \\
\hline ICA 5 -year change $(\mu \mathrm{m})$ & 0.06 & 0.43 & $0 \cdot 12$ & 0.41 & $0 \cdot 10$ & 0.37 & 0.08 & 0.34 & 0.7690 \\
\hline ICA percentage change (\%) & 8.97 & 43.38 & 13.79 & 43.69 & 16.98 & $54 \cdot 26$ & 11.31 & $43 \cdot 24$ & 0.4563 \\
\hline
\end{tabular}

PAI-1, plasminogen activator inhibitor 1 ; $S_{1}$, insulin sensitivity; AIR, acute insulin response; CCA, common carotid artery; IMT, intimal medial thickness; ICA, internal carotid artery.

*Simplification conducted using method 1 (stepwise linear regression on $Y$ score, $\alpha=0.05$ criteria) applied to first food pattern score obtained in reduced rank procedure.

Based on previous work, sex, race/ethnicity, impaired glucose tolerance status and baseline obesity status were considered $a$ priori as potential effect modifiers of the association and evaluated by stratification and by including pattern score by covariate interaction terms. No evidence for effect modification was found. All analyses were performed in SAS (version 8.2; SAS Institute, Inc., Cary, NC, USA).

\section{Results}

Table 1 illustrates characteristics of the original full food pattern score and its relation with the nine food groups constituting the primary simplified food pattern score (using method 1, M1). The food groups' low-fibre bread and cereal and red and processed meat explained $21 \cdot 2$ and $16 \cdot 1 \%$, respectively,

Table 3. Measures of subclinical atherosclerosis and 5-year change according to quartile of food intake pattern score at baseline $(n 802)$

\begin{tabular}{|c|c|c|c|c|c|c|c|c|c|c|}
\hline & \multicolumn{4}{|c|}{ CCA IMT* } & \multicolumn{5}{|c|}{ ICA IMT* } & \multirow[b]{3}{*}{$P$ for trend } \\
\hline & \multicolumn{4}{|c|}{$\begin{array}{l}\text { Quartiles of simplified food } \\
\text { pattern score }\end{array}$} & \multirow[b]{2}{*}{$P$ for trend } & \multicolumn{4}{|c|}{$\begin{array}{l}\text { Quartiles of simplified } \dagger \text { food } \\
\text { pattern score }\end{array}$} & \\
\hline & 1 & 2 & 3 & 4 & & 1 & 2 & 3 & 4 & \\
\hline IMT mean at follow-up $(\mu \mathrm{m})$ & $0.79 \neq \S$ & $0.82 \|$ & $0.87 \ddagger \|$ & $0.85 \S$ & 0.0032 & 0.95 & 1.02 & 1.03 & 1.01 & 0.2654 \\
\hline IMT difference $(\mu \mathrm{m})$ & $-0.02 \ddagger \S$ & $0.02 \|$ & $0.07 \ddagger \|$ & $0.06 \S$ & 0.0017 & 0.04 & 0.11 & 0.12 & 0.10 & 0.1982 \\
\hline Percentage change in IMT (\%) & $0.8 \pm \S$ & $3.2 \|$ & $8.6 \pm \|$ & $7.9 \S$ & 0.0045 & $7.2 \ddagger$ & 13.5 & $17.6 \neq$ & 13.7 & 0.2165 \\
\hline
\end{tabular}

CCA, common carotid artery; IMT, intimal medial thickness; ICA, internal carotid artery.

${ }^{*}$ All results are estimated from multiple linear regression models adjusted for age, sex, race/ethnicity, clinic, glucose tolerance status at baseline, smoking, systolic and diastolic blood pressure, BMI and total and HDL-cholesterol.

† Method 1: stepwise linear regression on $Y$ score, $\alpha=0.05$ criteria applied to first pattern score obtained in reduced rank procedure.

$¥ 1$ v. 3 significant at the 0.05 level.

$\S 1 \mathrm{v} .4$ significant at the 0.05 level.

|l 2 v. 3 significant at the 0.05 level. 
Table 4. Impact of simplification methods on association of dietary pattern score with subclinical atherosclerosis $(n$ 802) ( $\beta$ Values with their standard errors)

\begin{tabular}{|c|c|c|c|c|c|c|c|}
\hline \multirow[b]{2}{*}{ Simplification method } & \multirow[b]{2}{*}{ Total food groups } & \multicolumn{3}{|c|}{ CCA IMT* } & \multicolumn{3}{|c|}{ ICA IMT† } \\
\hline & & $\beta(\mu m)$ & $\mathrm{SE}(\mu \mathrm{m})$ & $P$ & $\beta(\mu \mathrm{m})$ & $\mathrm{SE}(\mu \mathrm{m})$ & $P$ \\
\hline Full, original scoreł & 33 & 0.020 & 0.007 & 0.007 & 0.036 & 0.015 & 0.02 \\
\hline Method $1 \S$ & 9 & 0.006 & 0.002 & 0.01 & 0.006 & 0.005 & 0.20 \\
\hline Method 2\| & 10 & 0.004 & 0.002 & 0.05 & 0.004 & 0.004 & 0.20 \\
\hline Method 39 & 10 & 0.005 & 0.002 & 0.03 & 0.007 & 0.005 & 0.14 \\
\hline Method $4^{\star *}$ & 9 & 0.003 & 0.002 & 0.08 & 0.005 & 0.004 & 0.17 \\
\hline
\end{tabular}

CCA, common carotid artery; IMT, intimal medial thickness; ICA, internal carotid artery.

${ }^{\star}$ Results are adjusted for age, sex, race/ethnicity/clinic, glucose tolerance status at baseline, smoking, systolic and diastolic blood pressure, BMI, total and HDL-cholesterol and baseline CCA.

†Results are adjusted for age, sex, race/ethnicity/clinic, glucose tolerance status at baseline, smoking, systolic and diastolic blood pressure, BMI, total and HDL-cholesterol and baseline ICA.

$\ddagger$ The food pattern score presented here is the first score obtained in the reduced rank procedure.

$\S$ Method 1: stepwise linear regression on $Y$ score of either CCA or ICA, $\alpha=0.05$ criteria (food groups: low-fibre bread and cereal; rice and pasta; tomato foods; cottage cheese; red and processed meat; poultry; soft drinks and lemonades; meal replacements; wine).

|| Method 2: stepwise linear regression on $Y$ score of either CCA or ICA, ten food groups with highest explained variation (food groups: low-fibre bread and cereal; red and processed meat; cottage cheese; wine; tomato foods; regular soft drinks and lemonades; cheese; rice and pasta; meal replacements; salty snacks).

I Method 3: stepwise linear regression on $Y$ score of either CCA or ICA, five positively, five inversely associated food groups with highest explained variation (food groups: low-fibre bread and cereal; red and processed meat; cottage cheese; tomato foods; regular soft drinks; wine; rice and pasta; meal replacements; poultry; high-fibre bread and cereal).

${ }^{\star *}$ Method 4: factor loading $\geq 0.2$ criteria (food groups: low-fibre bread and cereal; tomato foods; fried potatoes; cottage cheese; cheese; dried beans; eggs; red and processed meat; wine).

of the variation in the pattern score. Taken together, all nine food groups explained $81.5 \%$ of food pattern score variation. With increasing quartile of the simplified pattern score, the intake of less healthful food groups increased (low-fibre bread and cereal, red and processed meat, cottage cheese, tomato foods, regular soft drinks and sweetened beverages), while the intake of seemingly more healthful foods decreased (wine, rice and pasta, meal replacements and poultry).

Both PAI-1 and fibrinogen exhibited a positive association with the food pattern score (response score coefficients PAI-1 $=0.962$ and fibrinogen $=0.273$ ). The pattern score explained $8.7 \%$ of the variation in the inflammatory response variables and was largely driven by explained variation in PAI-1 (16\%) and marginally by fibrinogen ( $1 \%$; data not shown).

At baseline, marked associations between simplified food pattern score and age and race/ethnicity were observed (Table 2). By design of the RRR method, concentrations of PAI-1 and fibrinogen increased systematically with increasing food pattern score. Furthermore, BMI, waist, fasting insulin, acute insulin response, systolic and diastolic blood pressure, TAG and total cholesterol increased with increasing score quartile (Q) while $S_{\text {I }}$ and HDL-cholesterol were inversely related. As expected, mean CCA and ICA IMT levels increased over the 5 years for participants in all diet pattern quartiles.

A positive association between the simplified food pattern score and measures of CCA IMT was observed (Table 3). Mean CCA IMT at follow-up increased significantly across quartiles of food pattern score $(P=0.0032)$, flattening at Q3. The association was independent of age, sex, race/ethnicity, clinic, glucose tolerance status at baseline, smoking, systolic and diastolic blood pressure, BMI, total and HDL-cholesterol and baseline CCA IMT. Compared to individuals in the lowest quartile whose CCA IMT decreased slightly over 5 years (difference $=-0.02 \mu \mathrm{m}$ ), those in the highest quartile experienced a $0.06 \mu \mathrm{m}$ increase in absolute CCA wall thickness. The percentage change in CCA IMT increased across the first three food pattern quartiles: Q1 $0.8 \%$; Q2 3.2\%;
Q30 8.6\%; Q4 7.9\%. While the mean ICA wall thickness also seemed to increase across food pattern quartiles, the test for trend were not statistically significant. Of note, the percentage change in ICA was of larger magnitude than the one observed for CCA. No interactions with obesity, glucose tolerance, sex or race/ethnicity were observed. Additional adjustment for $S_{\mathrm{I}}$ or secretion, hypertension or lipid lowering medication, education or energy intake had no impact on findings for either CCA or ICA. Likewise, adjusting for waist circumference instead of BMI had no impact. Additional adjustment for 5-year change in CVD risk factors did not influence the results, hence only the results from the most parsimonious model are shown here.

Furthermore, we compared a total of four different simplification approaches with respect to their properties and impact on the association of interest in a type of sensitivity analysis (Table 4). The number of food groups selected from the original thirty-three was either nine or ten, of which five food groups were common to all methods and eight food groups common to three methods (M1, M2 and M3). The correlation between the full food pattern score (FS) and each of the simplified scores varied: $r_{\mathrm{FS} v . \mathrm{M} 1}=0.31$ $(P=0.0001) ; \quad r_{\mathrm{FS} v . \mathrm{M} 2}=0.55 \quad(P=0.0001) ; \quad r_{\mathrm{FS} v . \mathrm{M} 3}=0.21$ $(P=0.0001) ; \quad r_{\mathrm{FS} v . \mathrm{M} 4}=0.68 \quad(P=0.0001)$. However, with respect to the food intake score-IMT relationship, all methods identified a significant positive association with mean CCA IMT at follow-up, independent of age, sex, race/ethnicity, clinic, glucose tolerance status at baseline, smoking, systolic and diastolic blood pressure, BMI, total and HDL-cholesterol and baseline CCA IMT. Furthermore, regardless of which simplification method was used, results with respect to ICA IMT were similar in showing a lack of a significant association with the simplified score.

\section{Discussion}

We identified a food pattern that was strongly predictive of change in CCA IMT over a 5-year period in a cohort of 
middle-aged adults, using PAI-1 and fibrinogen as response variables and the RRR method. With increasing quartile of this food pattern, intake of low-fibre bread and cereal, red and processed meat, cottage cheese, tomato foods, regular soft drinks and sweetened beverages increased and intake of wine, rice and pasta, meal replacements and poultry decreased. Initially, identifying tomato foods among the less healthful food pattern constituents seemed counterintuitive given known beneficial properties of tomatoes. However, it is important to recognise that the FFQ question on tomato and tomato juice intake was explicitly phrased to include tomatoes consumed chopped or as condiments, which would often pertain to hamburger dishes. Furthermore, upon closer evaluation of the composition of the aggregate tomato food group, it seems likely that in our population, tomatoes are frequently consumed with other high-fat foods in the context of meals such as spaghetti, lasagna or pizza and that the unhealthful components may have overpowered the health-promoting properties of the tomato foods. Likewise, the identification of cottage cheese and ricotta intake as part of the less healthful patterns was initially perplexing, but we have previously shown that intake of this food group is associated with intake of many other less healthful food items in our population ${ }^{(17)}$. Finally, it should be noted that meal replacements (which included instant breakfasts and other liquid meals) were an item that was reported by participants in the open foods section of the interview and hence were included in the analysis because they were consumed at least once a week.

Several other investigators have identified similar food intake patterns associated with cardiovascular risk. In a recent analysis of the Whitehall-II cohort, McNaughton et al. ${ }^{(4)}$ demonstrated that high consumption of white bread, burgers and sausages, fried potatoes, sugar in tea and coffee and soft drinks and low consumption of French dressing and vegetables were associated with a $50 \%$ increase in risk of CHD. Hoffmann et al. ${ }^{(3)}$ reported that higher intakes of meat, poultry, margarine, other vegetable fats and oils and sauces and low intake of vegetarian dishes, cooked and raw vegetables, wine and whole grain cereals were associated with a markedly increased risk of CHD in a case-control study. Only one prospective study to date has identified a largely healthful food intake pattern associated with decreased risk of CHD, which comprised increased intakes of whole grain bread, fresh fruit, olive oil, mushrooms, cruciferous vegetables, wine and nuts and decreased intake of fried potatoes ${ }^{(2)}$.

Of note, previous research has varied with respect to the biomarkers selected as response variables ${ }^{(2-4,25)}$. We too initially evaluated a panel of blood lipids comparable to the Whitehall-II Study ${ }^{(4)}$ and other traditional cardiovascular risk factors including blood pressure and anthropometric characteristics and measures of $S_{\mathrm{I}}$ but found that only the selection of PAI-1 and fibrinogen as response variables yielded dietary patterns that were informative in our study population.

The present study is, to the best of our knowledge, the first prospective study using RRR to identify dietary predictors of precursors of CHD, i.e. changes in subclinical atherosclerosis. We have found a strong association between dietary intake and progression of CCA IMT. The integrative nature of RRR, rather than the previous focus on single or few nutrients or food items, sheds light on the etiologic process that seems to involve pro-inflammatory and pro-thrombotic pathways and impacts the development of atherosclerosis long before clinical symptoms appear. While persons in the top three quartiles of the food intake score experienced an increase in CCA wall thickness, those in the lowest quartile, i.e. the most healthful range of the dietary pattern, experienced a small decrease in CCA wall thickness over the 5-year period, independent of baseline risk factor levels.

A previous cross-sectional study using RRR to derive patterns from C-reactive protein, IL-6, homocysteine and fibrinogen concentrations identified a pattern that was quite different from ours. Furthermore, the pattern score was not significantly associated with either CCA or ICA IMT after adjustment for traditional CVD risk factors, although the association with CCA IMT was noteworthy and just shy of statistical significance $^{(26)}$. Interestingly, the strong association observed in our data was limited to CCA IMT but not with progression of ICA IMT. This finding is consistent with a previous work in IRAS in which we have reported a significant (inverse) association between whole grain intake with CCA IMT progression, while the association with ICA progression did not reach statistical significance ${ }^{(27)}$. Furthermore, in IRAS, CCA and ICA progression are not strongly correlated ${ }^{(28)}$. Also, in the Multi-Ethnic Study of Atherosclerosis, which used a very similar protocol for diet assessment and subclinical atherosclerosis assessment, a recent cross-sectional analysis reported a significant, albeit weaker inverse association between a largely detrimental food intake pattern and CCA IMT and a lack of an association with ICA IMT ${ }^{(29)}$. Among potential explanations for the differences between CCA and ICA results are that CCA and ICA wall thickness measures may differentially reflect wall thickening $v$. formation of raised atherosclerotic plaque ${ }^{(19)}$. In the context of our findings, this could suggest that while there is a relation of food intake with wall thickening, there is no such relation with atherosclerotic plaque. Compared to the CCA measurement, the variability in ICA measurement was much higher which may be a reflection of the thickness of raised plaque overwhelming any contribution of the ICA intima and media. In toto, these effects may have reduced our ability to find a significant relationship with ICA IMT, even though the trend in our data is suggestive of a positive relation mirroring the food intake pattern-CCA IMT association.

The present study also included a sensitivity analysis to evaluate the extent to which the dietary pattern simplification methodology might impact the results. Simplification methods are useful, because the food intake scores originally identified by RRR do not have an immediate clinical interpretation because they are weighted linear functions of all standardised food groups included in the analysis (e.g. in the present study thirty-three food groups created from a 114-item FFQ). Schulze et al. ${ }^{(24)}$ first proposed the concept of a simplified pattern and conducted a comparative evaluation of the properties of the original $v$. a simplified score in the context of a factor analysis. Since then, several other data reduction approaches have been utilised ${ }^{(3,23,30)}$. However, to the best of our knowledge, none have evaluated the impact of the simplification method on their results. Simplification entails an inherent loss of information, in that only a reduced number of food groups are retained and that the weighting factors are removed. Our evaluation of four simplified scores revealed that the method is quite robust as each of the methods yielded 
similar results with respect to identifying the presence of a strong association with CCA IMT. While it is clear that the selected food groups may differ between the various simplification methods, a core group of five food groups which included low-fibre bread and cereal, tomato foods, cottage cheese, red and processed meat and wine intake was common to all methods. This common group might be an appealing starting point for intervention researchers or health practitioners interested in crafting a simple recommendation for a cardioprotective diet; however, other current guidelines need to be considered ${ }^{(31)}$.

A number of strengths and limitations are worth mentioning. The present study is one of the few ${ }^{(2,4)}$ prospective cohort studies to focus on RRR-determined dietary patterns and atherosclerosis as previous research has largely relied on case-control designs ${ }^{(2,3,25)}$. In the present study, the outcome of interest - carotid IMT - was assessed following a standardised protocol at baseline and follow-up. However, our sample size was quite modest when compared to others ${ }^{(4)}$. Even though the dietary intake reflects the usual intake over the past year, its assessment occurred contemporaneously with the collection of baseline levels of PAI-1 and fibrinogen. Therefore, we are unable to conclusively disentangle the temporal relationship between food intake and biomarkers. Finally, as previously described ${ }^{(27)}$, the IRAS cohort oversampled persons with impaired glucose tolerance because of its focus on questions related to $S_{\mathrm{I}}$. Thus, the present results may not be entirely generalisable to a lower risk general population. In this context, it is particularly encouraging that despite using a different set of biomarkers as response variables, our diet pattern is similar to that found in the Whitehall-II Study and similarly associated with CVD risk ${ }^{(4)}$.

In conclusion, we have identified a pro-inflammatory and pro-thrombotic dietary pattern that was positively associated with progression in the common carotid intima medial thickness in a middle-aged, multi-ethnic cohort. This association was independent of all traditional CVD risk factors as well as measures of $S_{\text {I }}$ and secretion. The pattern was characterised by higher intakes of low-fibre bread and cereal, red and processed meat, cottage cheese, tomato foods, regular soft drinks and sweetened beverages and lower intake of wine, rice and pasta, meal replacements and poultry. Five of these food groups (low-fibre bread and cereal, red and processed meat, cottage cheese, tomato foods, wine) were identified by all simplification methods evaluated. Persons in the most healthful range of the dietary pattern (i.e. the low end of the distribution) experienced a small decrease in CCA wall thickness over the 5 years, while all others experienced a thickening of the CCA wall. As our research demonstrates, RRR is a promising and robust tool that can more comprehensively address the complexity of dietary intake than previous approaches focused on single nutrients or foods. Because people generally consume foods in combinations, we contend that RRR is a particularly useful method among a larger set of diet pattern tools that may help us advance our knowledge of diet-health associations.

\section{Acknowledgements}

A. D. L. conceptualised the paper, designed the analytic approach, provided oversight to data management and analyses, interpreted the data and drafted and revised the manuscript. M. N. implemented the statistical analyses and contributed to the revision of the manuscript. D. H. contributed to the revision of the manuscript. P. B. M. contributed to the drafting and revision of the manuscript. M. S. provided guidance to the implementation of statistical analysis and contributed to manuscript drafting and revision. D. C. G. contributed to the drafting and revision of the manuscript. R. B. D. provided significant consultation on the statistical analysis plan and the interpretation of the results. The present study was supported by an American Heart Association Grant-in-Aid to Dr Liese. The IRAS study was supported by NIH/NHLBI grants UO1 HL/17 887, UO1 HL/17 889, UO1 HL/17 890, UO1 HL/17 892, UO1 HL/17 902 and DK29867. The authors have no financial or personal conflicts of interest to report. The supplementary material for this article can be found at http://www.journals.cambridge.org/bjn

\section{References}

1. Hoffmann K, Schulze MB, Schienkiewitz A, et al. (2004) Application of a new statistical method to derive dietary patterns in nutritional epidemiology. Am J Epidemiol 159, 935-944.

2. Weikert C, Hoffmann K, Dierkes J, et al. (2005) A homocysteine metabolism-related dietary pattern and the risk of coronary heart disease in two independent German study populations. J Nutr 135, 1981-1988.

3. Hoffmann K, Zyriax BC, Boeing H, et al. (2004) A dietary pattern derived to explain biomarker variation is strongly associated with the risk of coronary artery disease. Am J Clin Nutr 80, 633-640.

4. McNaughton SA, Mishra GD \& Brunner EJ (2009) Food patterns associated with blood lipids are predictive of coronary heart disease: the Whitehall II Study. Br J Nutr 102, 619-624.

5. Lorenz MW, Markus HS, Bots ML, et al. (2007) Prediction of clinical cardiovascular events with carotid intima-media thickness: a systematic review and meta-analysis. Circulation 115, 459-467.

6. Ernst E \& Resch KL (1993) Fibrinogen as a cardiovascular risk factor: a meta-analysis and review of the literature. Ann Intern Med 118, 956-963.

7. Folsom AR, Aleksic N, Park E, et al. (2001) Prospective study of fibrinolytic factors and incident coronary heart disease: the Atherosclerosis Risk in Communities (ARIC) Study. Arterioscler Thromb Vasc Biol 21, 611-617.

8. Aleksic N, Wang YW, Ahn C, et al. (2008) Assessment of coronary heart disease risk by combined analysis of coagulation factors. Atherosclerosis 198, 294-300.

9. Wagenknecht LE, Mayer EJ, Rewers M, et al. (1995) The Insulin Resistance Atherosclerosis Study (IRAS) objectives, design, and recruitment results. Ann Epidemiol 5, 464-472.

10. Bergman RN, Finegood DT \& Ader M (1985) Assessment of insulin sensitivity in vivo. Endocr Rev 6, 45-86.

11. Yang YJ, Youn JH \& Bergman RN (1987) Modified protocols improve insulin sensitivity estimation using the minimal model. Am J Physiol 253, 6 Pt 1, E595-E602.

12. Pacini G \& Bergman RN (1986) MINMOD: a computer program to calculate insulin sensitivity and pancreatic responsivity from the frequently sampled intravenous glucose tolerance test. Comput Methods Programs Biomed 23, 113-122.

13. Saad MF, Anderson RL, Laws A, et al. (1994) A comparison between the minimal model and the glucose clamp in the assessment of insulin sensitivity across the spectrum of glucose tolerance. Insulin Resistance Atherosclerosis Study. Diabetes 43, 1114-1121. 
14. World Health Organization (1985) Diabetes Mellitus: Report of a WHO Study Group. Technical Report Series no. 727. Geneva: World Health Organization.

15. Festa A, D'Agostino R Jr, Tracy RP, et al. (2002) Elevated levels of acute phase proteins and plasminogen activator inhibitor-1 (PAI-1) predict the development of type 2 diabetes mellitus: the Insulin Resistance Atherosclerosis Study (IRAS). Diabetes 51, 1131-1137.

16. Mayer-Davis EJ, Vitolins MZ, Carmichael SL, et al. (1999) Validity and reproducibility of a food frequency interview in a Multi-Cultural Epidemiology Study. Ann Epidemiol 9, 314-324.

17. Liese AD, Schulz M, Moore CG, et al. (2004) Dietary patterns, insulin sensitivity, and adiposity in the multi-ethnic Insulin Resistance Atherosclerosis Study population. Br J Nutr 92, 973-984.

18. Mayer-Davis EJ, D'Agostino R Jr, Karter AJ, et al. (1998) Intensity and amount of physical activity in relation to insulin sensitivity: the Insulin Resistance Atherosclerosis Study. JAMA 279, 669-674.

19. Touboul PJ, Hennerici MG, Meairs S, et al. (2007) Mannheim carotid intima-media thickness consensus (2004-2006). An update on behalf of the Advisory Board of the 3rd and 4th Watching the Risk Symposium, 13th and 15th European Stroke Conferences, Mannheim, Germany, 2004, and Brussels, Belgium, 2006. Cerebrovasc Dis 23, 75-80.

20. O'Leary DH, Polak JF, Wolfson SK Jr, et al. (1991) Use of sonography to evaluate carotid atherosclerosis in the elderly. The Cardiovascular Health Study. CHS Collaborative Research Group. Stroke 22, 1155-1163.

21. The ARIC Study Group (1991) High-resolution B-mode ultrasound reading methods in the Atherosclerosis Risk in Communities (ARIC) cohort. J Neuroimaging 1, 168-172.

22. The ACAPS Group (1992) Rationale and design for the Asymptomatic Carotid Artery Plaque Study (ACAPS). Control Clin Trials 13, 293-314.
23. Schulze MB, Hoffmann K, Manson JE, et al. (2005) Dietary pattern, inflammation, and incidence of type 2 diabetes in women. Am J Clin Nutr 82, 675-684.

24. Schulze MB, Hoffmann K, Kroke A, et al. (2003) An approach to construct simplified measures of dietary patterns from exploratory factor analysis. Br J Nutr 89, 409-419.

25. DiBello JR, Kraft P, McGarvey ST, et al. (2008) Comparison of 3 methods for identifying dietary patterns associated with risk of disease. Am J Epidemiol 168, 1433-1443.

26. Nettleton JA, Steffen LM, Schulze MB, et al. (2007) Associations between markers of subclinical atherosclerosis and dietary patterns derived by principal components analysis and reduced rank regression in the Multi-Ethnic Study of Atherosclerosis (MESA). Am J Clin Nutr 85, 1615-1625.

27. Mellen PB, Liese AD, Tooze JA, et al. (2007) Whole-grain intake and carotid artery atherosclerosis in a multiethnic cohort: the Insulin Resistance Atherosclerosis Study. Am J Clin Nutr 85, 1495-1502.

28. Espeland MA, Evans GW, Wagenknecht LE, et al. (2003) Site-specific progression of carotid artery intimal-medial thickness. Atherosclerosis 171, 137-143.

29. Nettleton JA, Schulze MB, Jiang R, et al. (2008) A priori-defined dietary patterns and markers of cardiovascular disease risk in the Multi-Ethnic Study of Atherosclerosis (MESA). Am J Clin Nutr 88, 185-194.

30. Heidemann C, Hoffmann K, Spranger J, et al. (2005) A dietary pattern protective against type 2 diabetes in the European Prospective Investigation into Cancer and Nutrition (EPIC)-Potsdam Study cohort. Diabetologia 48, 1126-1134.

31. Lichtenstein AH, Appel LJ, Brands M, et al. (2006) Diet and lifestyle recommendations revision 2006: a scientific statement from the American Heart Association Nutrition Committee. Circulation 114, 82-96. 